\title{
Lorentz-Violating Electrodynamics and the Cosmic Microwave Background
}

\author{
V. Alan Kostelecký ${ }^{1}$ and Matthew Mewes ${ }^{2}$ \\ ${ }^{1}$ Physics Department, Indiana University, Bloomington, Indiana 47405, USA \\ ${ }^{2}$ Physics Department, Marquette University, Milwaukee, Wisconsin 53201, USA
}

(Received 13 February 2007; published 3 July 2007)

\begin{abstract}
Possible Lorentz-violating effects in the cosmic microwave background are studied. We provide a systematic classification of renormalizable and nonrenormalizable operators for Lorentz violation in electrodynamics and use polarimetric observations to search for the associated violations.
\end{abstract}

Relativity has been confirmed to a high degree of precision by many experiments over the past century. Recent years have seen renewed interest in sensitive tests of relativity following the realization that tiny violations of Lorentz symmetry, which forms the basis of both Special and General Relativity, can arise in theories that attempt to unify all known forces [1]. While contemporary searches for Lorentz violations involve many types of matter and energy, the properties of light have traditionally been the primary focus. Today, searches for dominant relativityviolating effects involving photons include modern versions of the classic Michelson-Morley and KennedyThorndike experiments [2-7] and analyses of polarized light from distant astrophysical sources [8,9]. The latter take advantage of the extreme propagation times over which tiny effects can accumulate, and they yield sensitivities comparable to those achieved with matter [10-12]. The cosmic microwave background (CMB), which is the oldest untainted radiation available to observation, offers a unique opportunity for Lorentz-violation searches involving photons. In this Letter, we introduce a systematic classification of coefficients for Lorentz violation at all orders, develop theoretical tools to extract sensitivity from polarimetric observations of the CMB, and analyze observational data to obtain first measurements of various relativity-violating effects.

At attainable scales, Lorentz violations are described by the Standard-Model Extension (SME) [13]. The SME is an effective field theory that serves as the general theoretical basis for experimental searches, including ones with light. It categorizes the type of Lorentz violation by the mass dimension $d$ of the corresponding operator in the Lagrange density, which offers a simple measure of their expected size [14]. Existing studies of the SME photon sector primarily focus on operators of renormalizable dimension $d \leq 4$, but here we consider terms with arbitrary $d$ that preserve the usual U(1) and spacetime-translation symmetries and hence conserve charge, energy, and momentum. Some calculation reveals that in this case the photon sector of the SME Lagrange density takes the form

$$
\begin{aligned}
\mathcal{L}= & -\frac{1}{4} F_{\mu \nu} F^{\mu \nu}+\frac{1}{2} \epsilon^{\kappa \lambda \mu \nu} A_{\lambda}\left(\hat{k}_{A F}\right)_{\kappa} F_{\mu \nu} \\
& -\frac{1}{4} F_{\kappa \lambda}\left(\hat{k}_{F}\right)^{\kappa \lambda \mu \nu} F_{\mu \nu},
\end{aligned}
$$

where $A_{\mu}$ is the electromagnetic 4-potential and $F_{\mu \nu}$ is the field-strength tensor. The first term in $\mathcal{L}$ is conventional Maxwell electrodynamics, while the other terms violate Lorentz symmetry. The quantities $\left(\hat{k}_{A F}\right)_{\kappa}$ and $\left(\hat{k}_{F}\right)^{\kappa \lambda \mu \nu}$ are polynomials in the 4-momentum operator $p_{\mu}=i \partial_{\mu}$ given by

$$
\begin{aligned}
\left(\hat{k}_{A F}\right)_{\kappa} & =\sum_{d \text { odd }}\left(k_{A F}^{(d)}\right)_{\kappa}{ }^{\alpha_{1} \ldots \alpha_{(d-3)}} \partial_{\alpha_{1}} \ldots \partial_{\alpha_{(d-3)}}, \\
\left(\hat{k}_{F}\right)^{\kappa \lambda \mu \nu} & =\sum_{d \text { even }}\left(k_{F}^{(d)}\right)^{\kappa \lambda \mu \nu \alpha_{1} \ldots \alpha_{(d-4)}} \partial_{\alpha_{1}} \ldots \partial_{\alpha_{(d-4)}},
\end{aligned}
$$

where $k_{A F}^{(d)}, k_{F}^{(d)}$ are constant coefficients for Lorentz violation of dimension $4-d$. The coefficients $k_{A F}^{(d)}$ violate $C P T$ symmetry, while the coefficients $k_{F}^{(d)}$ preserve it. If these coefficients emerge from spontaneous breaking, the associated Nambu-Goldstone modes might play the role of the photon [15], but this issue is secondary and disregarded here. Note that relaxing U(1) invariance would introduce a $d=2$ photon-mass term, among other effects.

The operators in Eqs. (2) produce changes in the properties of electromagnetic radiation. The plane-wave solutions to the equations of motion obtained from Eq. (1) reveal that in the presence of Lorentz violation, light propagating in empty space can be viewed as a superposition of two modes differing in polarization and velocity. The difference in phase velocity between the modes causes a shift in the relative phase between the two modes during propagation, which alters the superposition and thereby produces cosmic birefringence. For each type of operator causing birefringence, the size of the effect is governed by the associated coefficient for Lorentz violation multiplied by a factor of $E^{d-3} t$, where $E$ is the photon energy and $t$ is the propagation time. For cosmological sources, this factor can become very large, providing extreme sensitivity to minuscule violations of Lorentz invariance. 
The CMB radiation is now known to be partially polarized [16-20] and has propagated for approximately $14 \times$ $10^{9}$ years, so even minuscule Lorentz violations could alter its polarization in a detectable way [21,22]. For CMB radiation, taking the observed photon energy as $E_{\mathrm{ob}} \sim$ $10^{-13} \mathrm{GeV}$ and the propagation time as $t \sim 10^{10} \mathrm{yr} \sim$ $10^{42} \mathrm{GeV}^{-1}$, we obtain a crude estimated sensitivity of parts in $10^{81-13 d} \mathrm{GeV}^{4-d}$ to dimension- $d$ coefficients for Lorentz violation. Since the sensitivity to relativity violations grows roughly as $E^{d-3}$, higher photon energies generally lead to higher sensitivities. One might therefore expect studies of the lower-energy microwaves in the CMB to yield lesser sensitivities than prior searches for birefringence from SME operators with $d=4$ performed using near-optical emissions from distant galaxies and gamma rays from gamma-ray bursts [9]. However, a significant advantage arises from the cosmological redshift. Much of the polarization change occurred shortly after the CMB was produced, when the Universe was much hotter and the photons were approximately 1000 times more energetic. This implies that studying the CMB is effectively equivalent to an optical test with a time scale set by some fraction of the Hubble time. Indeed, explicitly integrating the $\mathrm{CMB}$ energy from the time of last scattering to the present reveals that for operators with $d>5$, the effective sensitivity to Lorentz violation is well approximated by parts in $10^{67-10 d} /(d-5) \mathrm{GeV}^{4-d}$, a substantial improvement over the crude estimate. Table I provides numerical values of the integral and estimated sensitivities.

To study the implications of Lorentz violation for the observed CMB polarization, we must first understand the effects on the Stokes parameters $Q, U, V$ and the corresponding Stokes vector $s=\left(s^{1}, s^{2}, s^{3}\right)^{T}=(Q, U, V)^{T}$. The birefringence induced from Eq. (1) causes the Stokes vector $s$ characterizing the net polarization of the light to rotate about an axis given by the Stokes vector $\boldsymbol{s}$ for the faster mode [9]. The angle of rotation of $s$ is the change in relative phase. For a convenient normalization of $\boldsymbol{s}$, the differential rotation is given at leading order by

$$
d \boldsymbol{s} / d t=2 E \mathbf{s} \times \boldsymbol{s} \equiv-i \Sigma \cdot \boldsymbol{s},
$$

where $\Sigma$ is a matrix. The components of $\boldsymbol{s}$ and hence of $\Sigma$ control completely the polarization change as light propagates from a distant source to Earth, and they depend on coefficients for Lorentz violation, the photon frequency, and the propagation direction.
For given values of the coefficients for Lorentz violation, the change in polarization as light propagates is determined by integration of Eq. (3) from emission to detection. For the CMB radiation, the integration must be done for each point on the sky, and two issues must be addressed. The first is the cosmological redshift, which leads to decreasing photon frequencies and consequent changes in the rotation axis $\boldsymbol{S}$ as the light propagates. This typically makes analytical considerations sufficiently challenging that numerical integration is needed. The second issue involves the tensor nature of the Stokes parameters and the whole-sky nature of the CMB. For light propagating inward over the sphere of the sky, the Stokes parameters $s^{1}$ and $s^{2}$ are components of a symmetric 2-tensor in the tangent space of the sphere, while $s^{3}$ is a scalar. To obtain a global description capable of handling correlations in CMB data across the sky, it is convenient to work in a spin-weighted basis. By definition, a spin-weighted function ${ }_{s} f$ of weight $s$ transforms according to ${ }_{s} f^{\prime}=e^{-s \delta}{ }_{s} f$ under a local rotation by $\delta$ in the tangent space of the sphere. We define spinweighted Stokes parameters $s_{( \pm 2)}=s^{1} \mp i s^{2}$ of spinweight \pm 2 and $s_{(0)}=s^{3}$ of spin-weight 0 , and we adopt the spin-weighted basis in which the Stokes vector becomes $\boldsymbol{s}=\left(s_{(+2)}, s_{(0)}, s_{(-2)}\right)^{T}$. With these definitions, a global description can be achieved by decomposing various quantities of interest in terms of spin-weighted spherical harmonics ${ }_{s} Y_{l m}(\hat{n})[23,24]$. The ${ }_{s} Y_{l m}(\hat{n})$ can be viewed as the generalization of the usual spherical harmonics to tensors in the tangent space of the sphere, with integer indices restricted by $l \geq|s|$ and $m=-l, \ldots, l$. For a fixed spin weight $s$, the ${ }_{s} Y_{l m}(\hat{n})$ form a complete orthonormal set of spin- $s$ functions on the sphere.

The CMB temperature $T$ and the Stokes parameter $s^{3}=$ $s_{(0)}$ are scalars on the sphere and can be decomposed into the usual spherical harmonics $Y_{l m} \equiv{ }_{0} Y_{l m}$, while the Stokes parameters $s_{( \pm 2)}$ are combinations of harmonics with spin weight \pm 2 :

$$
\begin{gathered}
T=\sum_{l m} a_{(T) l m 0} Y_{l m}, \quad s_{(0)}=\sum_{l m} a_{(V) l m 0} Y_{l m}, \\
s_{( \pm 2)}=\sum_{l m}\left(a_{(E) l m} \pm i a_{(B) l m}\right)_{ \pm 2} Y_{l m} .
\end{gathered}
$$

Here, each amplitude obeys $a_{(X) l m}^{*}=(-1)^{m} a_{(X) l-m}$ with $X=T, E, B, V$. The notation $E$ and $B$ arises from the parity properties of the amplitudes, which mimics those of

TABLE I. CMB sensitivities to Lorentz-violating operators of dimension $d$. The first row lists numerical values of the energy integral in terms of $E_{\mathrm{ob}}$ and the Hubble constant $H_{0}=71 \mathrm{~km} / \mathrm{s} / \mathrm{Mpc}$. The second row gives the estimated sensitivity to the corresponding coefficient for Lorentz violation. The third row lists approximate sensitivities we obtain by comparison with B03 data. The cosmological parameters adopted in this work are $z_{\mathrm{CMB}}=1100, \Omega_{m}=0.27, \Omega_{\Lambda}=0.73, \Omega_{r}=0.015$.

\begin{tabular}{lccccc}
\hline \hline \multicolumn{1}{c}{$d$} & 3 & 4 & 5 & 6 & 7 \\
\hline$H_{0} \int\left(E / E_{\mathrm{ob}}\right)^{d-3} d t$ & 0.95 & 2.7 & 40 & $8.6 \times 10^{3}$ & $4.9 \times 10^{6}$ \\
Estimated sensitivity $\left(\mathrm{GeV}^{4-d}\right)$ & $10^{-42}$ & $10^{-29}$ & $10^{-18}$ & $10^{-7}$ & $10^{3}$ \\
B03 sensitivity $\left(\mathrm{GeV}^{4-d}\right)$ & $10^{-42}$ & $10^{-30}$ & $10^{-19}$ & $10^{-9}$ & $\cdots$ \\
\hline \hline
\end{tabular}


the electric and magnetic fields, while the notation $V$ arises from the Stokes usage for circular polarization. The above decompositions are convenient since general considerations predict the $\mathrm{CMB}$ has no $V$-type (circular) polarization and significant nonzero cross correlations only between the $T$ and $E$ amplitudes when reionization or other foreground effects are neglected [25]. Typically, CMB observations of temperature and polarization are expressed as estimates of power spectra and correlations via the coefficients $C_{l}^{X_{1} X_{2}} \equiv$ $\frac{1}{2 l+1} \sum_{m}\left\langle a_{\left(X_{1}\right) l m}^{*} a_{\left(X_{2}\right) l m}\right\rangle$. In the absence of Lorentz violation, a $T T$ component, a small $E E$ component, and a $T E$ correlation are predicted, consistent with existing data at present sensitivities. Even smaller $B B$ modes without $T B$ correlations are also expected, but confirmation of this lies beyond current observational reach.

The Stokes vector $\mathbf{s}$ determining the rotation axis for birefringence can also be decomposed in the spin-weighted basis, $\mathbf{s}=\left(\boldsymbol{s}_{(+2)}, \boldsymbol{s}_{(0)}, \boldsymbol{s}_{(-2)}\right)^{T}$. This gives

$$
\Sigma=2 E\left(\begin{array}{ccc}
\boldsymbol{\varsigma}_{(0)} & -\boldsymbol{\varsigma}_{(+2)} & 0 \\
-\frac{1}{2} \boldsymbol{S}_{(-2)} & 0 & \frac{1}{2} \boldsymbol{\varsigma}_{(+2)} \\
0 & \boldsymbol{\varsigma}_{(-2)} & -\boldsymbol{\varsigma}_{(0)}
\end{array}\right)
$$

The components $\boldsymbol{S}_{(s)}$ can be written explicitly in terms of the coefficients for Lorentz-violation in Eqs. (2). However, it is convenient here to expand in spin-weighted spherical harmonics. Some calculation yields

$$
\begin{aligned}
\mathbf{s}_{(0)} & =\sum_{d} \sum_{l m} E^{d-4} k_{(V) l m}^{(d)} Y_{l m}, \\
\mathbf{S}_{( \pm 2)} & =\sum_{d} \sum_{l m} E^{d-4}\left(k_{(E) l m}^{(d)} \pm i k_{(B) l m}^{(d)}\right)_{ \pm 2} Y_{l m} .
\end{aligned}
$$

Here, $d$ is odd for $\mathbf{s}_{(0)}, d$ is even for $\mathbf{s}_{( \pm 2)}, l \leq d-2$ for both, and $l \geq 2$ for $\boldsymbol{s}_{( \pm 2)}$. It follows that CMB Lorentz violations separate into three categories $E, B, V$ according to the operator dimension $d$ and its $P$ and $C P T$ properties. The coefficients for Lorentz violation $k_{(E) l m}^{(d)}, k_{(B) l m}^{(d)}, k_{(V) l m}^{(d)}$ are constants of dimension $E^{4-d}$. The $E, B$ effects preserve $C P T$, while the $V$ effects violate it.

A complete analysis of available CMB polarization data [16-20] is challenging because searching for Lorentz violation requires careful treatment of the frequency depen- dences. We avoid these complications here by focusing on results from the BOOMERANG (B03) experiment [16], which performed polarimetry in a single relatively narrow high-frequency band at approximately $145 \mathrm{GHz}$. The effects grow roughly as $E^{d-3}$, so inclusion of other lowerfrequency results may reduce errors but is unlikely to change sensitivities drastically. We match to the B03 data by comparing published values of $C_{l}$ [16] with those expected from nonzero birefringence. We assume conventional initial $C_{l}$, with nonzero $C_{l}^{T T}, C_{l}^{T E}$, and $C_{l}^{E E}$ only, calculated using available software [26]. The $T T$ data are unaffected by birefringence and can be disregarded here. Including them and varying the underlying cosmology or the initial $C_{l}$ to find the joint best-fit cosmological parameters and Lorentz-violating coefficients is expected to yield similar results because the $T T$ data dominate the statistics and our initial $C_{l}$ are consistent with other larger data sets. The initial $C_{l}$ are used to generate polarization maps of the sky. For chosen values of coefficients for Lorentz violation, the maps are propagated numerically via Eq. (3) to the present epoch, and the $C_{l}$ predicted today are extracted. For simplicity, we consider one nonzero coefficient at a time, although in principle, any combination of coefficients may exist in nature. The theoretical $C_{l}$ are binned to match the reported B03 values for $C_{l}^{T E}, C_{l}^{T B}, C_{l}^{E E}, C_{l}^{B B}$, and a $\chi^{2}$ distribution is constructed, $\chi^{2}=\sum_{\text {bins }}\left(C_{\mathrm{B} 03}-\right.$ $\left.C_{\text {theory }}\right)^{2} /\left(\sigma_{\text {B03 }}^{2}+\sigma_{\text {theory }}^{2}\right)$.

Figure 1 shows our estimated likelihoods for several types of Lorentz violations. The figure reveals that at the $1 \sigma$ level, the B03 data prefer nonzero values for all coefficients for Lorentz violation but are consistent with no violations at $2 \sigma$. Note that for $d>3$, the comparatively high B03 frequency leads to somewhat tighter constraints than our estimates in Table I, demonstrating the advantage of higher-energy studies. For each independent fit, the preferred values and $1 \sigma$ ranges of the coefficients are listed in Table II. Except for one special case, all coefficients for Lorentz violation cause either frequency- or directiondependent polarization rotations, resulting in complicated changes in polarization over the sky. Only coefficients with $d=3$ produce frequency-independent effects, and only the single special coefficient $k_{(V) 00}^{(3)}$ produces polarization rota-
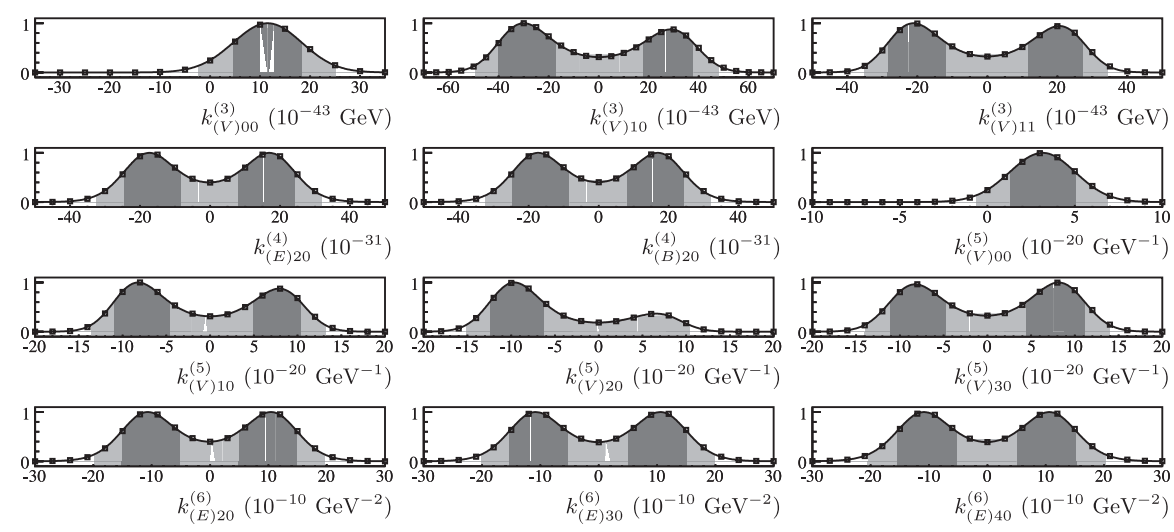


011601-3
FIG. 1. Sample plots of likelihood versus values of coefficients for Lorentz violation. For each listed coefficient, the boxes indicate numerically calculated values, and the curve is a smooth extrapolation through them. Dark-gray and light-gray regions represent the $68 \%$ and $95 \%$ confidence levels, respectively. 
TABLE II. Sample measured $1 \sigma$ values of coefficients showing $\chi^{2}$ per degree of freedom. Each fit is performed independently.

\begin{tabular}{lrc}
\hline \hline Coefficient & \multicolumn{1}{c}{ Value } & $\chi^{2} /$ d.o.f. \\
\hline$k_{(V) 00}^{(3)}$ & $(12 \pm 7) \times 10^{-43} \mathrm{GeV}$ & 1.2 \\
$k_{(V) 10}^{(3)}$ & $\pm(3 \pm 1) \times 10^{-42} \mathrm{GeV}$ & 1.2 \\
$k_{(V) 11}^{(3)}$ & $\pm\left(21_{-9}^{+7}\right) \times 10^{-43} \mathrm{GeV}$ & 1.2 \\
$k_{(E) 20}^{(4)}$ & $\pm\left(17_{-9}^{+7}\right) \times 10^{-31}$ & 1.2 \\
$k_{(B) 20}^{(4)}$ & $\pm\left(17_{-9}^{+7}\right) \times 10^{-31}$ & 1.2 \\
$k_{(V) 00}^{(5)}$ & $(3 \pm 2) \times 10^{-20} \mathrm{GeV}^{-1}$ & 1.2 \\
$k_{(V) 10}^{(5)}$ & $\left(8_{-3}^{+2}\right) \times 10^{-20} \mathrm{GeV}^{-1}$ & 1.2 \\
& $-\left(8_{-4}^{+3}\right) \times 10^{-20} \mathrm{GeV}^{-1}$ & 1.2 \\
$k_{(V) 20}^{(5)}$ & $-(10 \pm 3) \times 10^{-20} \mathrm{GeV}^{-1}$ & 1.1 \\
$k_{(V) 30}^{(5)}$ & $\left(8_{-4}^{+3}\right) \times 10^{-20} \mathrm{GeV}^{-1}$ & 1.2 \\
$k_{(E) 20}^{(6)}$ & $-(8 \pm 3) \times 10^{-20} \mathrm{GeV}^{-1}$ & 1.2 \\
$k_{(E) 30}^{(6)}$ & $\pm\left(11_{-5}^{+4}\right) \times 10^{-10} \mathrm{GeV}^{-2}$ & 1.2 \\
$k_{(E) 40}^{(6)}$ & $\pm\left(11_{-6}^{+5}\right) \times 10^{-10} \mathrm{GeV}^{-2}$ & 1.2 \\
\hline \hline
\end{tabular}

tions that are also uniform over the entire sky. A recent study of this special case [21] found that B03 and other CMB data favor a small nonzero rotation angle of $6^{\circ} \pm 4^{\circ}$, which in the present context is equivalent to the value $k_{(V) 00}^{(3)} \simeq(6 \pm 4) \times 10^{-43} \mathrm{GeV}$ and is compatible with the result in Table II. At the $95 \%$ confidence level, we obtain an upper limit of $k_{(V) 00}^{(3)} \lesssim 26 \times 10^{-43} \mathrm{GeV}$. This is consistent with the constraint $k_{(V) 00}^{(3)} \leqslant 40 \times 10^{-43} \mathrm{GeV}$ obtained from radio-galaxy polarimetry [8].

Table II also includes various results for frequency- and direction-dependent birefringence effects. We find that $2 \sigma$ constraints on the coefficients $k_{(V) 10}^{(3)}$ and $k_{(V) 11}^{(3)}$, which control anisotropic Lorentz violations for $d=3$, lie at the level of $10^{-42} \mathrm{GeV}$. Violations involving operators with $d=4$ are constrained to the $2 \sigma$ level of $10^{-30}$. This limit is consistent with the existing partial constraints on these coefficients of approximately $10^{-32}$ obtained from spectropolarimetry of galaxies and of approximately $10^{-37}$ obtained from gamma-ray bursts [9]. However, the pointsource nature of these previous results means that, while extremely sensitive, they only cover a limited portion of the coefficient space. Among all coefficients with $d=5,6$, only $k_{(V) 00}^{(5)}$ is direction independent. Our $2 \sigma$ constraint on this coefficient is consistent with studies of its effects in other contexts [27]. For the direction-dependent coefficients with $d=5$ and $d=6$ given in Table II, the measurements listed are the first obtained.

Overall, our results demonstrate that studies of the CMB polarization offer broad sensitivity to possible effects from all coefficients for Lorentz violation in electrodynamics.
While incorporation of additional available data is unlikely to increase significantly the net sensitivity, other CMB experiments may provide tests of the robustness of the $1 \sigma$ birefringent signals and determine whether they could be indicative of systematic effects or more conventional phenomena such as foregrounds. If the signals persist, existing and future high-resolution polarimetric data could determine which types of violations are preferred. Whatever the outcome, CMB polarimetry provides highly sensitive tests of spacetime symmetries with the potential to reveal signals of fundamental physics.

This work was supported in part by DOE Grant No. DEFG02-91ER40661, by NASA Grant No. NAG3-2194, and by the Wisconsin Space Grant Consortium.

[1] V. A. Kostelecký and S. Samuel, Phys. Rev. D 39, 683 (1989).

[2] J. A. Lipa et al., Phys. Rev. Lett. 90, 060403 (2003).

[3] H. Müller et al., Phys. Rev. Lett. 91, 020401 (2003).

[4] P. L. Stanwix et al., Phys. Rev. Lett. 95, 040404 (2005).

[5] S. Herrmann et al., Phys. Rev. Lett. 95, 150401 (2005).

[6] P. Antonini et al., Phys. Rev. A 71, 050101 (2005).

[7] P. L. Stanwix et al., Phys. Rev. D 74, 081101 (2006).

[8] S. M. Carroll et al., Phys. Rev. D 41, 1231 (1990).

[9] V. A. Kostelecký and M. Mewes, Phys. Rev. Lett. 87, 251304 (2001); Phys. Rev. D 66, 056005 (2002); Phys. Rev. Lett. 97, 140401 (2006).

[10] D. Bear et al., Phys. Rev. Lett. 85, 5038 (2000); M. A. Humphrey et al., Phys. Rev. A 68, 063807 (2003); F. Canè et al., Phys. Rev. Lett. 93, 230801 (2004).

[11] P. Wolf et al., Phys. Rev. Lett. 96, 060801 (2006).

[12] B. R. Heckel et al., Phys. Rev. Lett. 97, 021603 (2006).

[13] D. Colladay and V. A. Kostelecký, Phys. Rev. D 55, 6760 (1997); Phys. Rev. D 58, 116002 (1998); V. A. Kostelecký, Phys. Rev. D 69, 105009 (2004).

[14] V. A. Kostelecký and R. Potting, Nucl. Phys. B 359, 545 (1991); Phys. Rev. D 51, 3923 (1995).

[15] R. Bluhm and V. A. Kostelecký, Phys. Rev. D 71, 065008 (2005); B. Altschul and V. A. Kostelecký, Phys. Lett. B 628, 106 (2005).

[16] T.E. Montroy et al., Astrophys. J. 647, 813 (2006); F. Piacentini et al., Astrophys. J. 647, 833 (2006).

[17] E. M. Leitch et al., Astrophys. J. 624, 10 (2005).

[18] D. Barkats et al., Astrophys. J. 619, L127 (2005).

[19] A. C.S. Readnead et al., Science 306, 836 (2004).

[20] L. Page et al., avXiv:astro-ph/0603450.

[21] B. Feng et al., Phys. Rev. Lett. 96, 221302 (2006).

[22] A. Lue et al., Phys. Rev. Lett. 83, 1506 (1999); K. R. S. Balaji et al., J. Cosmol. Astropart. Phys. 12 (2003) 008; G.-C. Liu et al., Phys. Rev. Lett. 97, 161303 (2006).

[23] E. T. Newman and R. Penrose, J. Math. Phys. (N.Y.) 7, 863 (1966).

[24] J. N. Goldberg et al., J. Math. Phys. (N.Y.) 8, 2155 (1967).

[25] W. Hu and M. White, New Astron. Rev. 2, 323 (1997).

[26] U. Seljak and M. Zaldarriaga, Astrophys. J. 469, 437 (1996).

[27] T. A. Jacobson et al., Phys. Rev. Lett. 93, 021101 (2004); R. C. Myers and M. Pospelov, ibid. 90, 211601 (2003). 